\title{
Postoperative subdural hygroma and chronic subdural hematoma after unruptured aneurysm surgery: age, sex, and aneurysm location as independent risk factors
}

\author{
Jaechan Park, MD, PhD, ${ }^{1,2}$ Jae-Hoon Cho, MD, ${ }^{1}$ Duck-Ho Goh, MD, ${ }^{1}$ Dong-Hun Kang, MD, ${ }^{1,2}$ \\ Im Hee Shin, PhD, ${ }^{3}$ and In-Suk Hamm, MD, PhD'
}

${ }^{1}$ Department of Neurosurgery and ${ }^{2}$ Research Center for Neurosurgical Robotic Systems, Kyungpook National University, Daegu; and ${ }^{3}$ Department of Medical Statistics, School of Medicine, Catholic University of Daegu, Republic of Korea

\begin{abstract}
OBJECTIVE This study investigated the incidence and risk factors for the postoperative occurrence of subdural complications, such as a subdural hygroma and resultant chronic subdural hematoma (CSDH), following surgical clipping of an unruptured aneurysm. The critical age affecting such occurrences and follow-up results were also examined.

METHODS The case series included 364 consecutive patients who underwent aneurysm clipping via a pterional or superciliary keyhole approach for an unruptured saccular aneurysm in the anterior cerebral circulation between 2007 and 2013. The subdural hygromas were identified based on CT scans 6-9 weeks after surgery, and the volumes were measured using volumetry studies. Until their complete resolution, all the subdural hygromas were followed using CT scans every 1-2 months. Meanwhile, the CSDHs were classified as nonoperative or operative lesions that were treated by bur-hole drainage. The age and sex of the patients, aneurysm location, history of a subarachnoid hemorrhage (SAH), and surgical approach (pterional vs superciliary) were all analyzed regarding the postoperative occurrence of a subdural hygroma or CSDH. The follow-up results of the subdural complications were also investigated.
\end{abstract}

RESULTS Seventy patients (19.2\%) developed a subdural hygroma or CSDH. The results of a multivariate analysis showed that advanced age $(p=0.003)$, male sex $(p<0.001)$, middle cerebral artery $(M C A)$ aneurysm $(p=0.045)$, and multiple concomitant aneurysms at the MCA and anterior communicating artery $(A C O A)(p<0.001)$ were all significant risk factors of a subdural hygroma and CSDH. In addition, a receiver operating characteristic (ROC) curve analysis revealed a cut-off age of $>60$ years, which achieved a $70 \%$ sensitivity and $69 \%$ specificity with regard to predicting such subdural complications. The female patients $\leq 60$ years of age showed a negligible incidence of subdural complications for all aneurysm groups, whereas the male patients $>60$ years of age showed the highest incidence of subdural complications at $50 \%-100 \%$, according to the aneurysm location. The subdural hygromas detected $6-9$ weeks postoperatively showed different follow-up results, according to the severity. The subdural hygromas that converted to a CSDH were larger in volume than the subdural hygromas that resolved spontaneously $(28.4 \pm 16.8 \mathrm{ml}$ vs $59.6 \pm 38.4 \mathrm{ml}, p=0.003)$. Conversion to a CSDH was observed in $31.3 \%$ (5 of 16), $64.3 \%$ (9 of 14), and $83.3 \%$ (5 of 6 ) of the patients with mild, moderate, and severe subdural hygromas, respectively.

CONCLUSIONS Advanced age, male sex, and an aneurysm location requiring extensive arachnoid dissection (MCA aneurysms and multiple concomitant aneurysms at the MCA and ACOA) are all correlated with the occurrence of a subdural hygroma and CSDH after unruptured aneurysm surgery. The critical age affecting such an occurrence is 60 years. http://thejns.org/doi/abs/10.3171/2015.1.JNS14309

KEY WORDS chronic subdural hematoma; craniotomy; intracranial aneurysm; postoperative complications; subdural hygroma; vascular disorders

$\mathrm{W}$ ITH the rapid progress in neuroimaging techniques and current widespread performance of MR and CT angiography, the incidence of patients being identified with unruptured cerebral aneurysms and referred for surgical treatment and endovascular coil- ing is increasing. Moreover, advances in surgical techniques are minimizing postoperative complications. In a recent surgical series of unruptured aneurysms less than $10-15 \mathrm{~mm}$, the incidence of major surgical morbidity was less than $1 \% .^{14,20}$ Notwithstanding, the postoperative oc-

ABBREVIATIONS ACOA = anterior communicating artery; $\mathrm{CSDH}=$ chronic subdural hematoma; ICA = internal carotid artery; $\mathrm{MCA}=$ middle cerebral artery; PACS = picture-archiving and communication system; ROC = receiver operating characteristic; $\mathrm{SAH}=$ subarachnoid hemorrhage.

SUBMITTED February 10, 2014. ACCEPTED January 28, 2015.

INCLUDE WHEN CITING Published online August 14, 2015; DOI: 10.3171/2015.1.JNS14309. 
currence of a subdural hygroma and resultant chronic subdural hematoma (CSDH) is still common and sometimes troublesome. . $15,17,24^{2}$

Although most subdural hygromas resolve spontaneously, they can persist for a while and even convert to a CSDH. Such persistence of subdural hygromas can become more problematic in patients with medical conditions requiring antiplatelet or anticoagulant medication. Also, the conversion of a subdural hygroma to a CSDH is potentially serious and can require bur-hole drainage.

Accordingly, this study investigated the incidence and risk factors for the occurrence of subdural complications, such as a subdural hygroma and $\mathrm{CSDH}$, and attempted to determine the critical age affecting such occurrences and to elucidate the follow-up results.

\section{Methods}

\section{Patient Population}

After institutional review board approval, a retrospective review was conducted of all surgical cases of an unruptured, nongiant aneurysm in the anterior cerebral circulation clipped using a pterional or superciliary keyhole approach without an acute subarachnoid hemorrhage (SAH) that were treated between January 2007 and October 2013. The detailed surgical techniques of the superciliary keyhole approach were previously reported by the current authors. ${ }^{19,20}$ The medical records were reviewed to obtain relevant clinical information, and all the radiological data in this study were obtained using an electronic picture-archiving and communication system (PACS). Of a total of 376 patients, 12 were excluded due to the absence of regular CT scans 6-9 weeks postoperatively or appropriate follow-up CT scans.

\section{Diagnosis of Subdural Hygroma and CSDH}

Brain CT scans were routinely performed on Day 1, within 1 week (at discharge), and 6-9 weeks postoperatively. Additional CT scans were conducted in the case of any symptoms, such as a persistent headache, and followup images were obtained in the case of any subdural complications detected in the CT scans.

A subdural hygroma, which is the accumulation of CSF in the subdural space, was identified based on a CT finding of a density similar to that of CSF in the unilateral or bilateral frontoparietal region. The CT scans taken 6-9 weeks postoperatively were used to measure the volume of the subdural hygroma. These volumetry studies were performed using the CT scans taken on the axial planes with a section thickness of $5 \mathrm{~mm}$. PiViewSTAR (INFINITT Co., Ltd.), a PACS, was used to calculate the area of the subdural hygroma in each axial slice based on manually outlining the region of interest. The areas in each slice were then multiplied by the thickness of the slice and summed to arrive at the final measurement. A subdural hygroma was diagnosed in cases of a volume $>10 \mathrm{ml}$ or thickness $>4 \mathrm{~mm}$.

The severity of each subdural hygroma was arbitrarily classified as follows, using the CT scans taken 6-9 weeks postoperatively: mild subdural hygroma with a volume < $30 \mathrm{ml}$; moderate subdural hygroma with a volume 30-60 $\mathrm{ml}$; and severe, bilateral subdural hygroma with a volume
$>60 \mathrm{ml}$ (Fig. 1). The subdural hygromas were all followed up using CT scans every 1-2 months until their resolution.

A CSDH was diagnosed based on a CT finding of a crescent-shaped isodense or slightly hyperdense extraaxial collection in the frontoparietal region contrary to the hypodense subdural hygroma, and then classified as a nonoperative CSDH (asymptomatic CSDH $<1.5 \mathrm{~cm}$ thick with spontaneous resolution) or operative CSDH (symptomatic or thick CSDH treated by bur-hole drainage). Similarly, the CSDHs were all followed up using CT scans every 1-2 months until their resolution (either spontaneously or after surgical treatment).

The radiological diagnosis of subdural complications and volumetry studies of the subdural hygromas using CT scans taken 6-9 weeks postoperatively were performed by the readers (J.H.C. and D.H.G.), who were blinded to the clinical course of the patients.

\section{Statistical Analysis}

Statistical analyses were performed with the aid of commercially available statistics software (SPSS version 19.0; SPSS, Inc. and MedCalc version 6.2; MedCalc, Inc.). To investigate the risk factors of a postoperative subdural hygroma and $\mathrm{CSDH}$, uni- and multivariate analyses were performed. A chi-square analysis was used for the categorical variables (sex, history of SAH, location of the aneurysm, and surgical approach). A 2-sample Student t-test was used for the quantitative variable (age) to select the significant variables for inclusion in the binary multiple logistic regression statistical test used to determine the variables influencing the occurrence of subdural complications. A receiver operating characteristic (ROC) curve analysis was also performed to determine the best cut-off age affecting the occurrence of a subdural hygroma and CSDH. Meanwhile, Fisher's exact test was performed to compare the follow-up results for different grades of subdural hygroma. A 1-way ANOVA was used to compare the volumes of the subdural hygromas between the groups with and without conversion to a CSDH. Differences with $\mathrm{p}$ values less than 0.05 were considered to be statistically significant.

\section{Results \\ Clinical Characteristics}

The clinical characteristics of the 364 patients who underwent a craniotomy for an unruptured aneurysm in the anterior cerebral circulation and made regular postoperative follow-up visits are summarized in Table 1. With a mean age of 57.5 years (median 57 years, range 33-78), 246 patients were women and 118 were men. Fifty-four patients (14.8\%) had a history of an SAH caused by a concomitant aneurysm.

The patients were grouped according to the extent of arachnoid dissection required to expose and clip the aneurysm as follows: Group A, those patients with a single internal carotid artery (ICA) aneurysm $(n=80)$, including a superior hypophyseal artery aneurysm $(\mathrm{n}=2)$, posterior communicating artery aneurysm $(n=50)$, anterior choroidal artery aneurysm $(\mathrm{n}=18)$, ICA superior wall aneurysm $(n=5)$, and ICA bifurcation aneurysm $(n=5)$, as well 

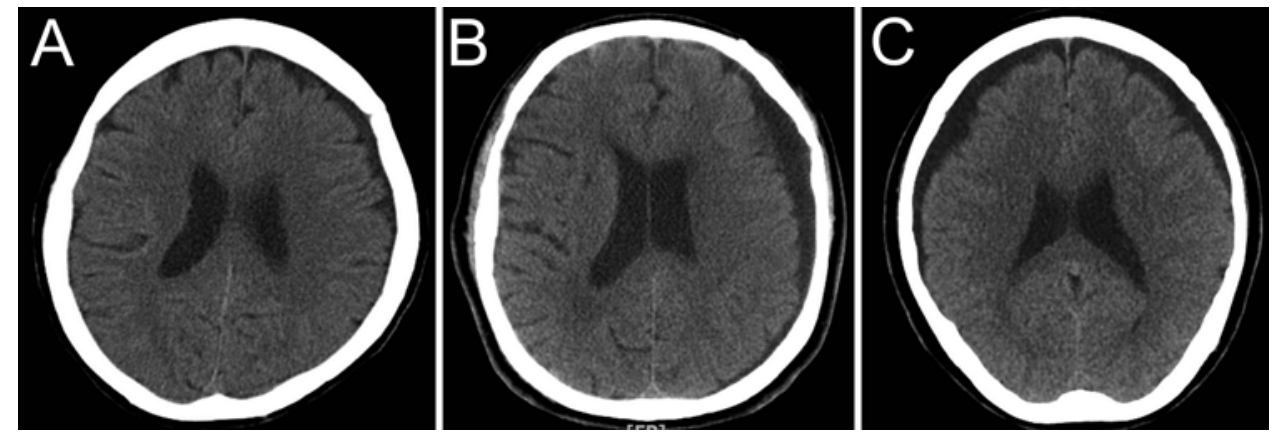

FIG. 1. Representative CT scans performed 6-9 weeks after unruptured aneurysm surgery showing mild (A), moderate (B), and severe (C) subdural hygromas.

as double ICA aneurysms $(n=3)$ and proximal $A_{1}$ aneurysm $(n=3)$; Group $B$, those patients with a single anterior communicating artery $(\mathrm{ACoA})$ aneurysm $(n=64)$, double ACoA aneurysms $(\mathrm{n}=5)$, and concomitant aneurysms (n =6) arising at the ICA and ACoA; Group C, those patients with a single middle cerebral artery (MCA) aneurysm ( $\mathrm{n}=$ 172) at the $M_{1}$ segment or MCA bifurcation, double MCA aneurysms $(n=4)$, and concomitant aneurysms $(n=13)$ arising at the ICA and MCA; Group D, those patients with concomitant aneurysms $(n=14)$ arising at the MCA and ACoA. A total of 409 aneurysms were clipped for the 364 patients.

Seventy-four patients (20.3\%) underwent a pterional craniotomy, and 290 patients $(79.7 \%)$ underwent a supraorbital mini-craniotomy using a $3.5-\mathrm{cm}$ eyebrow incision.

\section{Postoperative Subdural Hygroma}

Using the CT scans taken 6-9 weeks after surgery, subdural hygromas $>10 \mathrm{ml}$ were identified in 36 patients, the volumes ranging from 11 to $150 \mathrm{ml}$ (mean \pm SD: $44.9 \pm$ $33.8 \mathrm{ml}$ ). The subdural hygromas were classified as follows: mild $(n=16,19.5 \pm 6.0 \mathrm{ml})$, moderate $(n=14,47.4 \pm$ $10.4 \mathrm{ml})$, and severe $(\mathrm{n}=6,106.7 \pm 30.5 \mathrm{ml})$.

In the follow-up CT scans, all the subdural hygromas either resolved spontaneously or changed to a CSDH. In the latter case, the subdural hygromas on the CT scans 6-9 weeks postoperatively showed higher volumes than the subdural hygromas that resolved spontaneously ( 28.4 $\pm 16.8 \mathrm{ml}$ vs $59.6 \pm 38.4 \mathrm{ml}, \mathrm{p}=0.003)$. No subdural hygroma required bur-hole drainage without conversion to a large CSDH.

According to the severity of the subdural hygroma, a statistically significant difference was found in the incidence of spontaneous resolution, development of a nonoperative CSDH, and development of a CSDH requiring bur-hole drainage $(\mathrm{p}=0.009)$ (Fig. 2). The conversion of a subdural hygroma to a CSDH was observed in $31.3 \%$ (5 of 16), $64.3 \%$ (9 of 14), and 83.3\% (5 of 6) of patients with mild, moderate, and severe subdural hygromas, respectively, whereas all the remaining patients showed spontaneous resolution of the subdural hygroma.

\section{Postoperative CSDH}

A CSDH was diagnosed in 34 (9.3\%) of the 364 pa- tients. Of these 34 patients, the CSDH was minimal and resolved spontaneously in the follow-up CT scans in 17 patients (4.7\%), whereas the remaining 17 patients $(4.7 \%)$ required surgical treatment due to the volume of the CSDH being $>1.5 \mathrm{~cm}$ thick and/or related symptoms. The bur-hole drainage of a CSDH was performed 4-20 weeks (mean \pm SD: $9.1 \pm 3.8$ weeks, median 8 weeks) after the aneurysm surgery.

\section{Risk Factors of Subdural Hygroma and CSDH}

The univariate analysis revealed that male sex $(\mathrm{p}<$ $0.001)$, advanced age $(\mathrm{p}<0.001)$, and aneurysm location $(\mathrm{p}=0.003)$ were all potential risk factors for the postop-

TABLE 1. Clinical characteristics of 364 patients who underwent surgical clipping for unruptured aneurysms in anterior cerebral circulation

\begin{tabular}{lc}
\hline \multicolumn{1}{c}{ Characteristic } & No. of Patients (\%) \\
\hline Male & $118(32.4)$ \\
\hline Mean age in yrs, \pm SD & $57.5 \pm 10.1$ \\
\hline History of SAH & $54(14.8)$ \\
\hline Location of aneurysm & \\
\hline Group A & $86(23.6)$ \\
\hline Single ICA aneurysm & 80 \\
\hline Double ICA aneurysms & 3 \\
\hline Proximal A aneurysm & 3 \\
\hline Group B & $75(20.6)$ \\
\hline Single ACoA aneurysm & 64 \\
\hline Double ACoA aneurysms & 5 \\
\hline ICA aneurysm \& ACoA aneurysm & 6 \\
\hline Group C & $189(51.9)$ \\
\hline Single MCA aneurysm & 172 \\
\hline Double MCA aneurysms & 4 \\
\hline ICA aneurysm \& MCA aneurysm & 13 \\
\hline Group D & $14(3.8)$ \\
\hline ACoA aneurysm \& MCA aneurysm & 14 \\
\hline Surgical approach & $74(20.3)$ \\
\hline Pterional & $290(79.7)$ \\
\hline Superciliary &
\end{tabular}




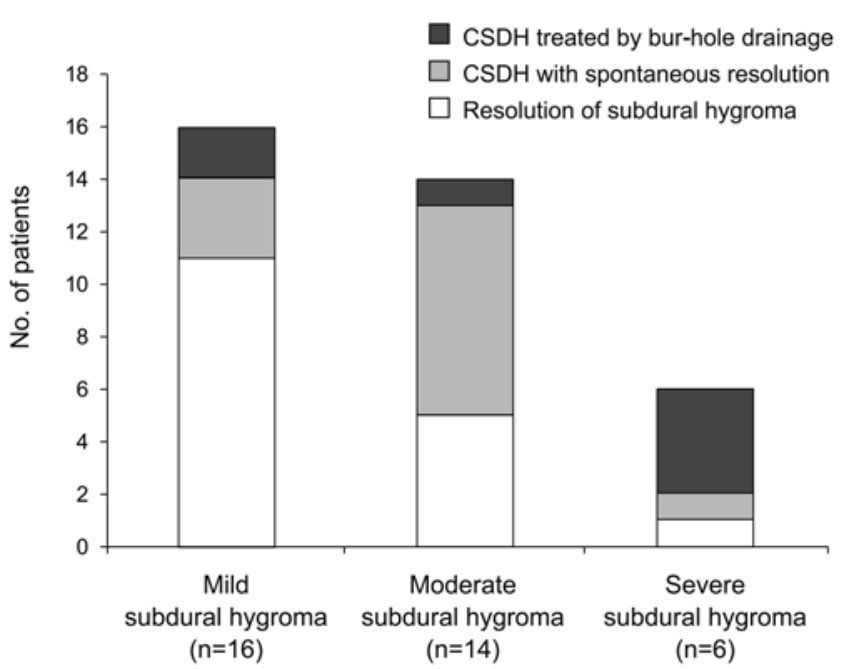

FIG. 2. Bar graph showing follow-up results for subdural hygromas observed 6-9 weeks after unruptured aneurysm surgery.

erative occurrence of subdural complications, such as a subdural hygroma and CSDH (Table 2). However, the surgical approach (pterional vs superciliary) and a history of SAH were not significant risk factors. Meanwhile, for the occurrence of an operative CSDH, male sex $(p<0.001)$ and advanced age $(\mathrm{p}=0.027)$ were identified as potential risk factors.

Based on the multivariate regression analysis, male sex (OR 3.697, 95\% CI 2.133-6.409, p < 0.001), advanced age (OR 0.988, 95\% CI 0.980-0.996, $\mathrm{p}=0.003)$, and aneurysm location including Group C (OR 2.183, 95\% CI
1.019-4.677, $\mathrm{p}=0.045)$ and Group D (OR 16.395, 95\% CI 4.233-63.499, $\mathrm{p}<0.001$ ) were all identified as risk factors of subdural complications (Table 3). Meanwhile, for the occurrence of an operative CSDH, male sex (OR 7.181, 95\% CI 2.852-18.077, $\mathrm{p}<0.001)$ and advanced age (OR $0.953,95 \%$ CI $0.939-0.967, \mathrm{p}<0.001)$ were the only risk factors (Table 4).

\section{Critical Age Affecting Occurrence of Subdural Hygroma and CSDH}

An ROC curve analysis for age was performed to determine the cut-off value for the occurrence of subdural complications, such as a subdural hygroma and CSDH. The results revealed that patient age $>60$ years was the appropriate cut-off value (area under the curve [AUC] 0.716, sensitivity $70.0 \%$, specificity 69.0\%) (Fig. 3).

\section{Incidence of Subdural Complications According to Known Risk Factors}

Based on the known risk factors including the critical age (60 years), sex, and aneurysm location, the incidence of subdural complications including subdural hygroma and CSDH is shown in Fig. 4. The female patients $\leq 60$ years of age exhibited a negligible incidence of subdural complications for all aneurysm groups, whereas the male patients $>60$ years of age showed the highest incidence of subdural complications at $50 \%$ (2 of 4 patients), $64 \%$ (9 of 14 patients), 67\% (8 of 12 patients), and $100 \%$ (3 of 3 patients) for Groups A, B, C, and D, respectively. Meanwhile, the incidence of subdural complications was approximately $32 \%$ (19 of 60 and 13 of 41 patients) for the female patients $>60$ years of age and male patients $\leq 60$

TABLE 2. Factors affecting occurrence of subdural hygroma or CSDH after surgery for unruptured aneurysms on univariate analysis

\begin{tabular}{|c|c|c|c|c|c|}
\hline \multirow[b]{2}{*}{ Factor } & \multicolumn{5}{|c|}{ No. of Patients (\%) } \\
\hline & Total & Subdural Hygroma \& CSDH & p Value & Surgically Treated CSDH & p Value \\
\hline Patients & 364 & $70(19.2)$ & & $17(4.7)$ & \\
\hline Sex & & & $<0.001^{*}$ & & $<0.001^{*}$ \\
\hline Female & 246 & $30(12.2)$ & & $2(0.8)$ & \\
\hline Male & 118 & $40(33.9)$ & & $15(12.7)$ & \\
\hline Mean age in yrs, \pm SD & $57.5 \pm 10.1$ & $63.5 \pm 10.0$ & $<0.001 \dagger$ & $62.0 \pm 11.0$ & $0.027 \dagger$ \\
\hline History of SAH & & & $0.852^{*}$ & & $0.742^{*}$ \\
\hline Absent & 310 & $60(19.4)$ & & $15(4.8)$ & \\
\hline Present & 54 & $10(18.5)$ & & $2(3.7)$ & \\
\hline Aneurysm location & & & $0.003^{*}$ & & $0.115^{*}$ \\
\hline Group A & 86 & $8(9.3)$ & & $1(1.2)$ & \\
\hline Group B & 75 & $14(18.7)$ & & $6(8.0)$ & \\
\hline Group C & 189 & $41(21.7)$ & & $9(4.8)$ & \\
\hline Group D & 14 & $7(50.0)$ & & $1(7.1)$ & \\
\hline Surgical approach & & & $0.431^{*}$ & & $0.136^{*}$ \\
\hline Pterional & 74 & $12(16.2)$ & & $2(2.7)$ & \\
\hline Superciliary & 290 & $58(20.0)$ & & $15(5.2)$ & \\
\hline
\end{tabular}


TABLE 3. Risk factors for occurrence of subdural hygroma or CSDH after surgery for unruptured aneurysms on multivariate analysis

\begin{tabular}{cccc}
\hline \multicolumn{1}{c}{ Variable } & OR & $95 \% \mathrm{Cl}$ & $\mathrm{p} \mathrm{Value}$ \\
\hline Sex & \multicolumn{3}{c}{} \\
\hline Female & 1 & $\mathrm{NA}$ & $\mathrm{NA}$ \\
\hline Male & 3.697 & $2.133-6.409$ & $<0.001$ \\
\hline Age & 0.988 & $0.980-0.996$ & 0.003 \\
\hline Aneurysm location & \multicolumn{3}{c}{} \\
\hline Group A & 1 & $\mathrm{NA}$ & $\mathrm{NA}$ \\
\hline Group B & 1.582 & $0.651-3.841$ & 0.311 \\
\hline Group C & 2.183 & $1.019-4.677$ & 0.045 \\
\hline Group D & 16.395 & $4.233-63.499$ & $<0.001$ \\
\hline History of SAH & 1.445 & $0.705-2.961$ & 0.315 \\
\hline Surgical approach & \multicolumn{3}{c}{} \\
\hline Pterional & 1 & $\mathrm{NA}$ & $\mathrm{NA}$ \\
\hline Superciliary & 1.135 & $0.587-2.193$ & 0.707 \\
\hline
\end{tabular}

NA = not applicable.

years of age, respectively, in Group C, including patients with an MCA aneurysm.

\section{Clinical Outcomes}

None of the patients with a subdural hygroma developed significant neurological deficits, although mild to moderate headache or fatigue was sometimes experienced for 1-2 weeks in the $2 \mathrm{nd}$ or $3 \mathrm{rd}$ week postoperatively. The mild subdural hygromas detected 6-9 weeks postoperatively were all resolved within 3-4 months after surgery, whereas it took 3-6 months after surgery for the moderate and severe subdural hygromas to be resolved.

Meanwhile, all the patients with a CSDH recovered without neurological sequelae after spontaneous resolution or surgical treatment. None of the patients experienced recurrence of the CSDH.

\section{Discussion}

For patients who undergo surgery for an unruptured aneurysm, previous studies have already identified advanced age and male sex as the risk factors for a CSDH.,15,17,24 In contrast, the present study investigated postoperative subdural complications, such as subdural hygromas and CSDHs, as a continuum and identified independent risk factors, including an aneurysm location requiring extensive dissection of the arachnoid membrane covering the sylvian fissure (MCA aneurysms, as well as multiple aneurysms at the MCA and ACoA), in addition to advanced age and male sex. Furthermore, aneurysms requiring extensive arachnoid dissection, including ACoA and MCA aneurysms, with the exception of ICA aneurysms, reached near significance $(0.05<\mathrm{p}<0.1)$ as risk factors for an operative CSDH in the multivariate analysis. The critical age affecting the occurrence of subdural complications was determined as $>60$ years. The present study also graded the subdural hygromas using CT scans taken 6-9 weeks
TABLE 4. Risk factors for occurrence of CSDH requiring bur-hole drainage after unruptured aneurysm surgery on multivariate analysis

\begin{tabular}{clcc}
\hline \multicolumn{1}{c}{ Variable } & OR & $95 \% \mathrm{Cl}$ & $\mathrm{p} \mathrm{Value}$ \\
\hline Sex & \multicolumn{1}{c}{$\mathrm{NA}$} & $\mathrm{NA}$ \\
\hline Female & 1 & $2.852-18.077$ & $<0.001$ \\
\hline Male & 7.181 & $0.939-0.967$ & $<0.001$ \\
\hline Age & 0.953 & & \\
\hline Aneurysm location & \multicolumn{1}{c}{$\mathrm{NA}$} & $\mathrm{NA}$ \\
\hline Group A & 1 & $0.961-23.253$ & 0.056 \\
\hline Group B & 4.727 & $0.836-17.043$ & 0.084 \\
\hline Group C & 3.774 & $0.822-58.247$ & 0.075 \\
\hline Group D & 6.919 & $0.337-3.117$ & 0.965 \\
\hline History of SAH & 1.025 & & \\
\hline Surgical approach & & $\mathrm{NA}$ & $\mathrm{NA}$ \\
\hline Pterional & 1 & $0.473-4.315$ & 0.527 \\
\hline Superciliary & 1.429 & &
\end{tabular}

after surgery to predict the duration of their persistence and the follow-up results according to the severity.

The occurrence of a subdural hygroma and resultant development of a CSDH have already been reported in patients who suffer traumatic brain injury or undergo neurosurgical procedures.11,16,18,21,27 One assumed mechanism leading to a subdural hygroma is the sequence of traumatic tearing or surgical dissection of the arachnoid membrane, the formation of a 1-way valve by adhesion of the arachnoid during the healing process, and the accumulation of CSF via the 1-way valve in the subdural space..$^{11,27}$

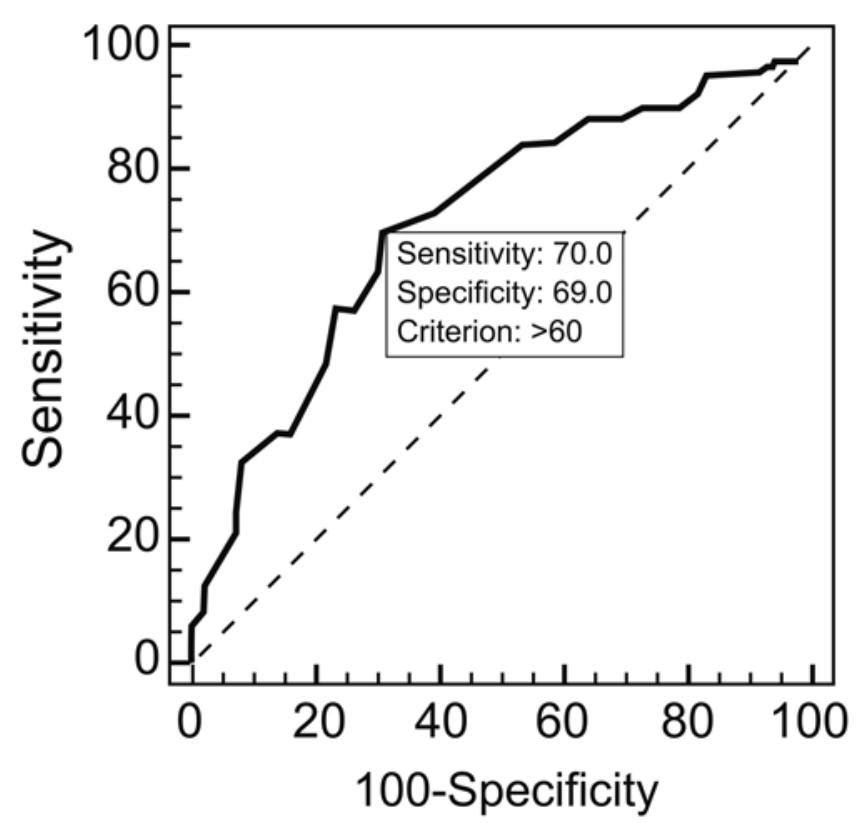

FIG. 3. ROC curve of patient age as a variable for predicting the occurrence of subdural hygroma and CSDH. AUC 0.716 , cut-off $>60$ years, sensitivity $70.0 \%$, specificity $69.0 \%$. 


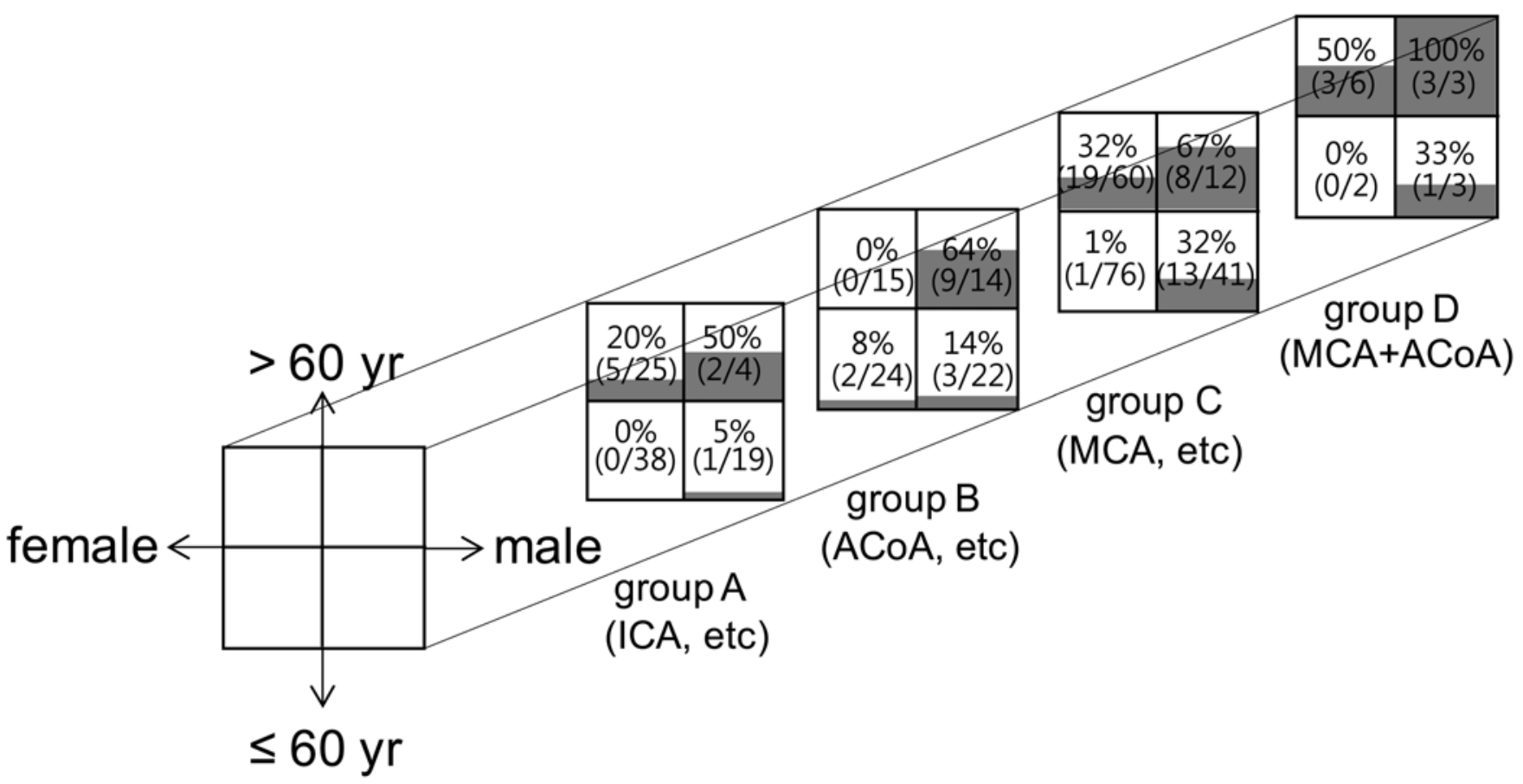

FIG. 4. Diagram demonstrating the incidence of subdural complications according to the age, sex, and aneurysm location.

Meanwhile, tearing of the stretched bridging veins and bleeding from the neomembrane of the CSDH are the suggested mechanisms for the development and enlargement of a CSDH.

Surgical clipping of unruptured aneurysms in the circle of Willis requires dissection of the arachnoid membrane covering the basal cisterns and sylvian fissure. Thereafter, the adhesive healing process facilitating the 1-way-valve phenomenon persists for a while. In particular, this seems to take longer for the sylvian fissure than the basal cisterns. The sylvian fissure, which is in a nondependent position, is widened following the dissection of the arachnoid, whereas the basal cisterns are located under the brain and the dissected gaps are reduced and covered by the undersurface of the brain after completion of the surgery.

Age-related brain atrophy shows decreased gray- and white-matter volumes and resultant increases in the CSF volumes in the sylvian fissure (frontotemporal atrophy), sulci (cortical atrophy), and ventricles (central cerebral atrophy). $4,6,12,22,23,25,28$ Such brain atrophy with aging occurs heterogeneously with regional differences. In the case of aneurysm surgery for elderly patients, the ensuing period of the 1-way-valve phenomenon for the dissected arachnoid can be prolonged due to the widened sylvian fissure and basal cisterns. This prolonged 1-way-valve phenomenon increases the degree and duration of a subdural hygroma. Furthermore, the reduced brain matter itself reduces the resistance against the subdural accumulation of CSF. Thus, the prolongation and severity of the subdural hygroma increases the risk of a CSDH.

The reasons a subdural hygroma and CSDH occur more frequently in male patients are not fully understood, yet a sex difference in brain atrophy is one reason to consider. Differences in the degree and location of brain atrophy are known to vary with sex, and the degree of brain atrophy has been reported to be greater in male subjects, including higher sulcal and sylvian fissure CSF volumes than in female subjects. ${ }^{2,3,5,10,26}$

The incidence of a postoperative CSDH seems to differ according to the neurosurgical procedure. Mori and Maeda ${ }^{15}$ reported the following incidences of CSDHs after neurosurgical procedures: $0.4 \%$ following the removal of a brain tumor, $2.4 \%$ after clipping an aneurysm, and $5.9 \%$ after superficial temporal artery-to-MCA anastomosis. Also, Ohno et al. ${ }^{17}$ reported a significantly higher incidence of CSDHs after surgery for unruptured aneurysms than after surgery for ruptured aneurysms. The brain swelling following an SAH is considered to decrease the subdural space and resist the accumulation of CSF.

After surgery for an unruptured aneurysm, most occurrences of a subdural hygroma and CSDH are delayed and cannot be predicted based on postoperative CT scans taken within 1 week. In the current series, only 2 patients with a CSDH underwent bur-hole drainage as early as 4 weeks postsurgery, while the remaining patients underwent burhole drainage 6-20 weeks after aneurysm surgery. Thus, delayed CT scans need to be performed 1-2 months after surgery to check for a subdural hygroma or CSDH.

In the case of a moderate or severe subdural hygroma, it can persist longer than 6 months after surgery and can develop into a CSDH any time before its complete resolution. Although the prognosis of a postoperative subdural hygroma is generally favorable, the risk of a CSDH and possible duration of a subdural hygroma should be taken into account when determining the treatment modality (surgical vs endovascular) and treatment timing for those patients with the risk factors for a subdural hygroma, including advanced age ( $>60$ years), male sex, and aneurysm location 
at the MCA. In particular, careful attention should be paid to cases of concomitant multiple aneurysms at the MCA and ACoA.

To prevent a postoperative subdural hygroma, positive results have already been reported on blocking the communication between the CSF space and the subdural space, where a fibrin adhesive is applied to seal the arachnoid defect in the sylvian fissure in the case of aneurysm surgery and the cortical defect in the case of transcortical transventricular procedures. ${ }^{1,8,13}$ However, an allergic reaction was reported after arachnoid plasty using a fibrin sealant, and the overall benefits and risks of such procedures have not yet been ascertained based on a large case series. ${ }^{9}$

The current study is limited, because it is based on a retrospective review of a case series from a single institution. However, the large number of patients and strict radiological follow-up of the subdural complications until their resolution allow for a high level of confidence in the identified risk factors and final outcomes. Notwithstanding, the application of different surgical techniques (conventional vs minimally invasive approaches) was not randomized and no closure technique for the arachnoid membrane was performed. Meanwhile, in any future study, the risk factors identified in the current study should also be analyzed to investigate the efficacy of closure of the arachnoid to prevent a subdural hygroma, and male patients $>60$ years of age who undergo surgical clipping for an unruptured MCA aneurysm should be the main subjects for arachnoid plasty.

\section{Conclusions}

Advanced age, male sex, and an aneurysm location requiring extensive arachnoid dissection (MCA aneurysms and, in particular, multiple concomitant aneurysms at the MCA and ACoA) were all correlated with the occurrence of a subdural hygroma and CSDH after unruptured aneurysm surgery. The critical age affecting such an occurrence was $>60$ years.

\section{References}

1. Al-Yamany M, Del Maestro RF: Prevention of subdural fluid collections following transcortical intraventricular and/or paraventricular procedures by using fibrin adhesive. J Neurosurg 92:406-412, 2000

2. Coffey CE, Lucke JF, Saxton JA, Ratcliff G, Unitas LJ, Billig B, et al: Sex differences in brain aging: a quantitative magnetic resonance imaging study. Arch Neurol 55:169-179, 1998

3. Cowell PE, Turetsky BI, Gur RC, Grossman RI, Shtasel DL, Gur RE: Sex differences in aging of the human frontal and temporal lobes. J Neurosci 14:4748-4755, 1994

4. Fotenos AF, Snyder AZ, Girton LE, Morris JC, Buckner RL: Normative estimates of cross-sectional and longitudinal brain volume decline in aging and AD. Neurology 64:1032-1039, 2005

5. Gur RC, Mozley PD, Resnick SM, Gottlieb GL, Kohn M, Zimmerman R, et al: Gender differences in age effect on brain atrophy measured by magnetic resonance imaging. Proc Natl Acad Sci U S A 88:2845-2849, 1991

6. Hedman AM, van Haren NE, Schnack HG, Kahn RS,
Hulshoff Pol HE: Human brain changes across the life span: a review of 56 longitudinal magnetic resonance imaging studies. Hum Brain Mapp 33:1987-2002, 2012

7. Inamasu J, Watabe T, Ganaha T, Yamada Y, Nakae S, Ohmi $\mathrm{T}$, et al: Clinical characteristics and risk factors of chronic subdural haematoma associated with clipping of unruptured cerebral aneurysms. J Clin Neurosci 20:1095-1098, 2013

8. Jung TY, Jung S, Jin SG, Jin YH, Kim IY, Kang SS, et al: Prevention of postoperative subdural fluid collections following transcortical transventricular approach. Surg Neurol 68:172-176, 2007

9. Kanazawa R, Sato S, Iwamoto N, Teramoto A: Allergic reaction following arachnoid plasty with a fibrin sealant. Neurol Med Chir (Tokyo) 50:608-610, 2010

10. Kaye JA, DeCarli C, Luxenberg JS, Rapoport SI: The significance of age-related enlargement of the cerebral ventricles in healthy men and women measured by quantitative computed X-ray tomography. J Am Geriatr Soc 40:225-231, 1992

11. Lee KS: The pathogenesis and clinical significance of traumatic subdural hygroma. Brain Inj 12:595-603, 1998

12. Long X, Liao W, Jiang C, Liang D, Qiu B, Zhang L: Healthy aging: an automatic analysis of global and regional morphological alterations of human brain. Acad Radiol 19:785-793, 2012

13. Mino Y, Hirashima Y, Hamada H, Masuoka T, Yamatani K, Takeda S, et al: Effect of arachnoid plasty using fibrin glue membrane after clipping of ruptured aneurysm on the occurrence of complications and outcome in the elderly patients. Acta Neurochir (Wien) 148:627-631, 2006

14. Moroi J, Hadeishi H, Suzuki A, Yasui N: Morbidity and mortality from surgical treatment of unruptured cerebral aneurysms at Research Institute for Brain and Blood VesselsAkita. Neurosurgery 56:224-231, 2005

15. Mori K, Maeda M: Risk factors for the occurrence of chronic subdural haematomas after neurosurgical procedures. Acta Neurochir (Wien) 145:533-540, 2003

16. Murata K: Chronic subdural hematoma may be preceded by persistent traumatic subdural effusion. Neurol Med Chir (Tokyo) 33:691-696, 1993

17. Ohno T, Iihara K, Takahashi JC, Nakajima N, Satow T, Hishikawa $\mathrm{T}$, et al: Incidence and risk factors of chronic subdural hematoma after aneurysmal clipping. World Neurosurg 80:534-537, 2013

18. Ohno K, Suzuki R, Masaoka H, Matsushima Y, Inaba Y, Monma S: Chronic subdural haematoma preceded by persistent traumatic subdural fluid collection. J Neurol Neurosurg Psychiatry 50:1694-1697, 1987

19. Park J, Kang DH, Chun BY: Superciliary keyhole surgery for unruptured posterior communicating artery aneurysms with oculomotor nerve palsy: maximizing symptomatic resolution and minimizing surgical invasiveness. J Neurosurg 115:700-706, 2011

20. Park J, Woo H, Kang DH, Sung JK, Kim Y: Superciliary keyhole approach for small unruptured aneurysms in anterior cerebral circulation. Neurosurgery 68 ( 2 Suppl Operative):300-309, 2011

21. Park SH, Lee SH, Park J, Hwang JH, Hwang SK, Hamm IS: Chronic subdural hematoma preceded by traumatic subdural hygroma. J Clin Neurosci 15:868-872, 2008

22. Resnick SM, Goldszal AF, Davatzikos C, Golski S, Kraut MA, Metter EJ, et al: One-year age changes in MRI brain volumes in older adults. Cereb Cortex 10:464-472, 2000

23. Resnick SM, Pham DL, Kraut MA, Zonderman AB, Davatzikos $\mathrm{C}$ : Longitudinal magnetic resonance imaging studies of older adults: a shrinking brain. J Neurosci 23:3295-3301, 2003

24. Tanaka Y, Mizuno M, Kobayashi S, Sugita K: Subdural fluid collection following craniotomy. Surg Neurol 27:353-356, 1987 
25. Tang Y, Whitman GT, Lopez I, Baloh RW: Brain volume changes on longitudinal magnetic resonance imaging in normal older people. J Neuroimaging 11:393-400, 2001

26. Xu J, Kobayashi S, Yamaguchi S, Iijima K, Okada K, Yamashita K: Gender effects on age-related changes in brain structure. AJNR Am J Neuroradiol 21:112-118, 2000

27. Yoshimoto Y, Wakai S, Hamano M: External hydrocephalus after aneurysm surgery: paradoxical response to ventricular shunting. J Neurosurg 88:485-489, 1998

28. Yue NC, Arnold AM, Longstreth WT Jr, Elster AD, Jungreis CA, O'Leary DH, et al: Sulcal, ventricular, and white matter changes at MR imaging in the aging brain: data from the cardiovascular health study. Radiology 202:33-39, 1997

\section{Disclosure}

The authors report no conflict of interest concerning the materi- als or methods used in this study or the findings specified in this paper.

\section{Author Contributions}

Conception and design: Park. Acquisition of data: Cho, Goh, Kang. Analysis and interpretation of data: Park, Shin. Drafting the article: Park. Reviewed submitted version of manuscript: Hamm, Park, Kang, Shin. Statistical analysis: Shin. Administrative/technical/material support: Park. Study supervision: Hamm, Park.

\section{Correspondence}

In-Suk Hamm, Department of Neurosurgery, Kyungpook National University Hospital, 50, Samduk 2-ga, Jung-gu, Daegu 700-721, Republic of Korea. email: ishamm@knu.ac.kr. 